\title{
Steady state free precession cardiovascular magnetic resonance: accuracy of left and right ventricular functional assessment in children
}

\author{
Jennifer A Bryant ${ }^{1,2}$, Keith Godfrey ${ }^{1,4}$, Mark A Hanson ${ }^{1,3}$, Lucy Davies ${ }^{4}$, Alison Fletcher ${ }^{2 *}$, Charles Peebles ${ }^{2}$ \\ From 16th Annual SCMR Scientific Sessions \\ San Francisco, CA, USA. 31 January - 3 February 2013
}

\section{Background}

Cardiovascular Magnetic Resonance (CMR) is recognised as the gold standard technique for the assessment of cardiovascular function in adults. In children, image quality is dependent on compliance with motion control and breath-hold instructions. Inability to maintain repeated breath-holds in a consistent phase of respiration can result in respiratory misregistration errors. There is limited data on the accuracy of CMR in children.

\section{Methods}

CMR was performed on 198 healthy 9 year old children as part of a study of developmental influences on cardiovascular structure and function. Contiguous steady state free precession cine images were acquired in the short axis (SA) plane. Data sets were analysed using a manual technique (Osirix) to calculate left ventricular (LV) and right ventricular (RV) stroke volumes (SV). Papillary muscles and trabeculae were excluded from the blood pool. LV and RV SVs were compared with flow data derived from phase contrast velocity flow mapping sequences through the aortic root and the main pulmonary artery respectively.

\section{Results}

There were good correlations between aortic flow and LV $\mathrm{SV}(\mathrm{r}=0.69, \mathrm{p}<0.0001, \mathrm{n}=198)$, and between pulmonary flow volume and RVSV $(\mathrm{r}=0.64, \mathrm{p}<0.0001, \mathrm{n}=189)$. Using the Bland Altman method the mean difference between LVSV and aortic flow was $10 \mathrm{ml}(95 \% \mathrm{CI} 9$ to $11 \mathrm{ml})$. Mean difference between RV SVs and MPA flow was $12 \mathrm{ml}$ (95\%CI 10.8 to $13.2 \mathrm{ml}$ ). There was a strong correlation

${ }^{2}$ Cardiothoracic Radiology, University Hospital Southampton NHS Foundation Trust, Southampton, UK

Full list of author information is available at the end of the article between aortic and pulmonary flow volumes $(r=0.87$, $\mathrm{p}<0.0001, \mathrm{n}=198$ ). The mean difference between aortic and MPA flow volumes was $3.4 \mathrm{ml}$.

\section{Conclusions}

Children at the age of 9 years can comply with multiple breath-hold requirements, allowing accurate assessment of cardiovascular function using steady state free precession CMR. Although less accurate than in adults, useful measurements can be obtained at this age for research studies as there is a good correlation between flow derived SVs and those derived from the SA cine stack.

\section{Funding}

This work was supported by funding from the British Heart Foundation and the National Institute for Health Research (NIHR Southampton Biomedical Research Centre).

\section{Author details \\ ${ }^{1} \mathrm{NIHR}$ Southampton Biomedical Research Centre, University of Southampton and University Hospital Southampton NHS Foundation Trust, Southampton, UK. ${ }^{2}$ Cardiothoracic Radiology, University Hospital Southampton NHS Foundation Trust, Southampton, UK. ${ }^{3}$ Institute of Developmental Sciences, University of Southampton, Southampton, UK. ${ }^{4}$ MRC Lifecourse Epidemiology Unit, Southampton, UK.}

Published: 30 January 2013

doi:10.1186/1532-429X-15-S1-T9

Cite this article as: Bryant et al: Steady state free precession

cardiovascular magnetic resonance: accuracy of left and right

ventricular functional assessment in children. Journal of Cardiovascular

Magnetic Resonance 2013 15(Suppl 1):T9.
C Biomed Central

(0) 2013 Bryant et al; licensee BioMed Central Ltd. This is an Open Access article distributed under the terms of the Creative Commons Attribution License (http://creativecommons.org/licenses/by/2.0), which permits unrestricted use, distribution, and reproduction in any medium, provided the original work is properly cited. 